hep-th/9911116

MRI-PHY/P991133

\title{
Universality of the Tachyon Potential
}

\author{
Ashoke Sen 1 \\ Mehta Research Institute of Mathematics \\ and Mathematical Physics \\ Chhatnag Road, Jhoosi, Allahabad 211019, INDIA
}

\begin{abstract}
Using string field theory, we argue that the tachyon potential on a D-brane antiD-brane system in type II string theory in arbitrary background has a universal form, independent of the boundary conformal field theory describing the brane. This implies that if at the minimum of the tachyon potential the total energy of the brane antibrane system vanishes in a particular background, then it vanishes in any other background. Similar result holds for the tachyon potential of the non-BPS D-branes of type II string theory, and the D-branes of bosonic string theory.
\end{abstract}

\footnotetext{
${ }^{1}$ E-mail: asen@thwgs.cern.ch, sen@mri.ernet.in
} 


\section{Contents}

\begin{tabular}{lll}
\hline & Introduction and Summary & 2 \\
\hline
\end{tabular}

2 Tachyon potential from open string field theory on the D-branes 5

3 Fate of the U(1) gauge field under tachyon condensation 13

\begin{tabular}{|lll}
\hline Tachyon potential in closed bosonic string theory & 18
\end{tabular}

\section{Introduction and Summary}

It has been argued on various general grounds that the condensation of the tachyon living on a configuration of coincident D-brane anti-D-brane pair produces a configuration which is indistinguishible from the vacuum where there are no branes [1, 2, 3, 过, 河. This requires that the sum of the tensions of the brane and the antibrane is exactly cancelled by the (negative) value of the tachyon potential at the minimum of the potential. There is however no direct evidence of this phenomenon, since there is no explicit knowledge of the tachyon potential, except that it has a maximum at the origin corresponding to negative mass $^{2}$ of the tachyon. The difficulty in studying the tachyon potential can be traced to the fact that the zero momentum tachyon is far off-shell, and hence is outside the scope of study of first quantized string theory which deals with only on-shell S-matrix elements.

In this paper we shall study some general properties of the tachyon potential using open string field theory, - a formalism particularly suited for the study of off-shell string theory [6, ]. In particular we show that the tachyon potential on the brane antibrane system is universal, independent of the particular boundary conformal field theory describing the D-brane, except for an overall multiplicative factor which is proportional to the tension of the brane-antibrane pair before tachyon condensation. Thus for example, the potential will be the same for flat D-branes, D-branes wrapped on various cycles of internal compact manifold, or D-branes in the presence of background metric and anti-symmetric tensor fields. A similar result holds for the tachyon potential on a single D-brane of bosonic string theory, or a single unstable non-BPS D-brane of type II string theory [8, 9, 10, 5 . Although this does not prove the conjecture that at the minimum of the potential the tension of the brane antibrane system is exactly cancelled by the tachyon potential, this 
shows that if the conjecture is valid for D-brane anti-D-brane system in one background, then it is also valid for D-brane anti-D-brane system in any other background.

Let us now be more specific about the analysis and the result of the paper. Section 2 of the paper is devoted to the analysis of the tachyon potential using open string field theory. As already mentioned, we shall be interested in a configuration containing a single D-brane in bosonic string theory, or a D-brane anti-D-brane pair or a single non-BPS D-brane in type II string theory. Some of the tangential directions of the D-brane(s) may be wrapped on some non-trivial cycles of an internal space. In general such a system of D-branes is described by a non-trivial boundary conformal field theory. In order to give a uniform treatment of all systems of this kind, we shall assume that all directions tangential to the D-brane are compact; this can be easily achieved by compactifying the non-compact directions tangential to the brane on a torus of large radii. Thus the resulting configuration can be viewed as a particle like object in the remaining non-compact directions, which we shall take to be a Minkowski space $e^{2}$ of dimension $(n+1)$. If we denote the space-like non-compact directions by $X^{i}(1 \leq i \leq n)$, and the time direction by $X^{0}$, then the total world-sheet theory will contain a set of free fields $X^{0}, X^{1}, \ldots X^{n}$ with Neumann boundary condition on $X^{0}$ and Dirichlet boundary condition on $X^{1}, \ldots X^{n}$, together with a nontrivial boundary conformal field theory (BCFT) of central charge $(25-n)$ describing the dynamics of the coordinates in the compact direction. The main objective of the paper will be to show that the tachyon potential is independent of this BCFT.

For simplicity we shall focus our attention on D-branes of bosonic string theory during most of the paper; so let us explain our results first in this context. We shall show that tachyon potential has the form:

$$
V(T)=M f(T)
$$

where $f(T)$ is a universal function of the tachyon field $T$ independent of the BCFT describing the D-brane, and $M$ is the mass of the D-brane at $T=0$, which can depend on the BCFT under consideration.f During this analysis we shall also arrive at a precise

\footnotetext{
${ }^{2}$ This restriction is due to a technical reason. We shall identify the mass of the D-brane as the coefficient of the $\frac{1}{2}\left(\dot{X}^{i}\right)^{2}$ term in the action, and for this purpose we need some directions in which the space-time is an ordinary Minkowski space-time.

${ }^{3}$ Throughout this paper all masses and energies will be measured in the closed string metric.

${ }^{4}$ In the convention that we shall choose, the mass of the D-brane is also independent of the BCFT. However it depends on the open string coupling constant, whose relation to the closed string coupling constant may depend on the details of the BCFT.
} 
definition of the tachyonic mode(s) and the tachyon potential. We choose the additive constant in $V(T)$ such that it vanishes at $T=0$. Thus the total energy of the D-brane for a given value of $T$ will be given by

$$
M+V(T)=M\{1+f(T)\}
$$

According to the conjecture of [11, 12, at some extremum $T_{0}$ of the tachyon potential the negative contribution of the tachyon potential exactly cancels the mass of the D-brane. Thus according to this conjecture

$$
1+f\left(T_{0}\right)=0
$$

Although our analysis does not provide a proof of this relation, the universality of the function $f(T)$ shows that if the relation holds for any of the D-branes of the bosonic string theory (say the D0-brane of the bosonic string theory in 26 dimensional Minkowski space), then it must hold for all D-branes in all possible compactifications of bosonic string theory.

An exactly similar result holds for the brane antibrane system of type II string theory. In this case $M$ denotes the total mass of the brane-antibrane system under consideration. The function $f(T)$ differs from the corresponding function in the bosonic string theory, but it is again universal in the sense that it does not depend on the details of the BCFT describing the brane antibrane system. The conjecture of ref. [1, 2] again requires $\{1+$ $f(T)\}$ to vanish at an extremum $T_{0}$ of $f(T)$. This time, however, supersymmetry of the background space-time requires that $T_{0}$ satisfying eq.(1.3) represents a global minimum of the potential.

Finally the result also holds for the non-BPS D-brane of type II string theory, with $M$ now representing the mass of the non-BPS D-brane.

According to the conjecture of [1, 2, 10, 11], at $T=T_{0}$ the D-brane of bosonic string theory, the brane antibrane system of type II string theory, or the non-BPS D-brane of type II string theory, is indistinguishible from the vacuum where there is no D-brane. Since the tachyon is neutral under the 'center of mass' U(1) gauge field living on the brane (brane antibrane system), a vev of the tachyon field does not break this $U(1)$ gauge symmetry. On the other hand the vacuum without any D-brane does not contain such a $U(1)$ gauge field. This poses a puzzle[13, 4, 14. In section 3 we show that the results of section 2 points to a possible way out of this puzzle. Using the universality 
of the tachyon potential, and the fact that $\left(1+f\left(T_{0}\right)\right)$ vanishes at $T_{0}$, we argue that at $T=T_{0}$, the effective action involving the center of mass $\mathrm{U}(1)$ gauge field does not contain any term without derivative of the gauge field strength. In particular it implies that the standard gauge kinetic term is absent. We conjecture that the effective action at $T=T_{0}$ is altogether independent of the gauge field, so that the gauge field behaves as an auxiliary field. This would explain the absence of a dynamical U(1) gauge field at $T=T_{0}$. Its equations of motion forces all states carrying the $\mathrm{U}(1)$ charge to disappear from the spectrum.尸

Finally in section 4 we discuss generalization of our results to closed bosonic string theory. We show that arguments similar to the one given in section 2 can be used to establish the universality of the tachyon potential in any compactification of the bosonic string theory. However, since there is no compelling reason to believe that there is a stable minimum of this potential, the significance of this result is not entirely clear.

Although our analysis establishes the universality of the tachyon potential, it does not tell us what this universal function is. Explicit analysis of the tachyon potential in open string theory with all Neumann boundary conditions was carried out in ref.[16]. Some properties of the tachyon potential on the brane antibrane system have been analyzed previously in refs.[17, 18, 19]. Attempts at deriving the explicit form of the tachyon potential using open string field theory have been made earlier in refs. [20]. Similar analysis for closed string tachyons were carried out in refs. [21, 22, 23]. Some aspects of the universality of the tachyon potential have been addressed earlier in ref. 24].

\section{Tachyon potential from open string field theory on the D-branes}

We shall use Witten's open string field theory[6, 7] to analyse the tachyon potential, but any other formulation of covariant open string field theory will also suffice[25]. Although the original version of this theory was formulated for open strings in flat space-time with Neumann boundary conditions in all directions, it can be easily generalized to describe open strings living on a D-brane. We use the language of [26], as reformulated in [27] for describing string field theory in arbitrary background field. We shall begin our discussion with open strings living on a D-brane in bosonic string theory; and later generalise it to

\footnotetext{
${ }^{5}$ The argument given in this section is an expanded version of the analysis already presented in 15 .
} 
brane-antibrane system or non-BPS D-branes in superstring theories.

As mentioned in the introduction, we compactify all the spatial directions tangential to the D-brane. Thus we are dealing with the dynamics of a particle with infinite number of degrees of freedom, described by a $(0+1)$ dimensional string field theory. Since string field theory corresponds to second quantized string theory, a point in the classical configuration space of string field theory corresponds to a specific quantum state of the first quantized theory. As was shown in [6], in order to describe a gauge invariant string field theory we must include the full Hilbert space of states of the first quantized open string theory including the $b$ and $c$ ghost fields, subject to the condition that the state must carry ghost number 1 . Here we are using the convention that $b$ carries ghost number $-1, c$ carries ghost number 1 , and the $\mathrm{SL}(2, \mathrm{R})$ invariant vacuum $|0\rangle$ carries ghost number 0 . We shall denote by $\mathcal{H}$ the subspace of the full Hilbert space carrying ghost number 1 . Let $|\Phi\rangle$ be an arbitrary state in $\mathcal{H}$, and $\Phi(x)$ be the local field (vertex operator) in the conformal field theory which creates this state $|\Phi\rangle$ out of the $\mathrm{SL}(2, \mathrm{R})$ invariant vacuum:

$$
|\Phi\rangle=\Phi(0)|0\rangle
$$

Since we are dealing with open string theory, $\Phi(x)$ lives on the boundary of the world sheet. We shall choose the convention that the world-sheet is the upper half plane, and its boundary is the real axis labelled by $x$.

The open string field theory action, which is a map from $\mathcal{H}$ to the space of real numbers, is given by

$$
\mathcal{S}=-\frac{1}{g_{o}^{2}}\left(\frac{1}{2}\left\langle\Phi\left|Q_{B}\right| \Phi\right\rangle+\frac{1}{3}\left\langle f_{1} \circ \Phi(0) f_{2} \circ \Phi(0) f_{3} \circ \Phi(0)\right\rangle\right) .
$$

Here $g_{o}$ is a constant denoting the open string coupling constant, $Q_{B}$ is the BRST charge constructed out of the ghost oscillators and the matter stress tensor, and \langle\rangle denotes correlation functions in the combined matter and ghost conformal field theory. The overall - sign in front of the action is a reflection of the fact that we are using Minkowski metric with signature $(-++\ldots+) . f_{1}, f_{2}$ and $f_{3}$ are three conformal transformations given by,

$$
\begin{aligned}
& f_{1}(z)=-e^{-i \pi / 3}\left[\left(\frac{1-i z}{1+i z}\right)^{2 / 3}-1\right] /\left[\left(\frac{1-i z}{1+i z}\right)^{2 / 3}+e^{i \pi / 3}\right], \\
& f_{2}(z)=F\left(f_{1}(z)\right), \quad f_{3}(z)=F\left(f_{2}(z)\right),
\end{aligned}
$$

where $F$ is an $\mathrm{SL}(2, \mathrm{R})$ transformation

$$
F(u)=-\frac{1}{1+u}
$$


$f_{i} \circ \Phi(0)$ denotes the conformal transform of $\Phi(0)$ by $f_{i}$. Thus for example if $\Phi$ denotes a dimension $h$ primary field, then $f_{i} \circ \Phi(0)=\left(f_{i}^{\prime}(0)\right)^{h} \Phi(f(0))$. For non-primary fields there will be extra terms involving higher derivatives of $f_{i}$. The inner product appearing in the first term of the action is defined as

$$
\langle\Phi \mid \Psi\rangle=\langle I \circ \Phi(0) \Psi(0)\rangle
$$

where $I$ denotes the $\mathrm{SL}(2, \mathrm{R})$ transformation $I(z)=-(1 / z)$. We shall choose the convention where $\alpha^{\prime}=1$, and the $\mathrm{SL}(2, \mathrm{R})$ invariant vacuum $|0\rangle$ is normalized as

$$
\left\langle 0\left|c_{-1} c_{0} c_{1}\right| 0\right\rangle=L,
$$

$L$ being the (infinite) length of the time interval over which the action is evaluated. (For the purpose of normalization we shall pretend that the time direction is compact with radius $L / 2 \pi$.) $c_{n}$ are the modes of the ghost field $c(z)$ defined through the relation $c(z)=\sum c_{n} z^{-n+1}$. In general we normalize the Fock vacuum $\left|k_{0}\right\rangle \equiv \exp \left(i k_{0} X^{0}(0)\right)|0\rangle$ with $X^{0}$ momentum $k_{0}$ as

$$
\left\langle k_{0}\left|c_{-1} c_{0} c_{1}\right| k_{0}^{\prime}\right\rangle=2 \pi \delta\left(k_{0}+k_{0}^{\prime}\right),
$$

with the understanding that $\delta(0)$ is defined to be $L / 2 \pi$.

The equations of motion of string field theory are obtained by demanding that the variation of $\mathcal{S}$ with respect to $|\Phi\rangle$ vanishes. We can get the component form of the equations by decomposing $|\Phi\rangle$ in a complete set of basis states in $\mathcal{H}$, and setting to zero the variation of $\mathcal{S}$ with respect to each coefficient in this expansion.

The zero momentum tachyonic state of open string theory can be identified as

$$
c_{1}|0\rangle,
$$

created by the vertex operator $c(0)$ acting on $|0\rangle$. It is however clear that due to the cubic coupling in the string field theory action (2.2), once we switch on tachyon vacuum expectation value (vev), various other fields must also be switched on in order to satisfy the string field theory equations of motion. However, not all the fields need to be switched on. Suppose we can decompose $\mathcal{H}$ into two subspaces $\mathcal{H}_{1}$ and $\mathcal{H}_{2}$ such that $\mathcal{S}$ is always quadratic or higher order in the components of $|\Phi\rangle$ along the basis vectors of $\mathcal{H}_{2}$. If we now take $|\Phi\rangle$ to lie solely in $\mathcal{H}_{1}$, then all the equations of motion obtained by varying $\mathcal{S}$ 
with respect to the components of $|\Phi\rangle$ along $\mathcal{H}_{2}$ are automatically satisfied. Thus we can obtain a consistent truncation of the theory by restricting $|\Phi\rangle$ to $\mathcal{H}_{1}$ and evaluating $\mathcal{S}$ for this $|\Phi\rangle$. A solution of the equations of motion obtained by varying the truncated action with respect to comoponents of $|\Phi\rangle$ along $\mathcal{H}_{1}$ can automatically be regarded as a solution of the equations of motion of the full string field theory.

We shall now describe such a decomposition of $\mathcal{H}$. We include in $\mathcal{H}_{1}$ all states of ghost number 1 , obtained from the $\mathrm{SL}(2, \mathrm{R})$ invariant vacuum by the action of the ghost oscillators $b_{n}$ and $c_{n}$, and the Virasoro generators of the entire matter conformal field theory. In the language of vertex operators this will amount to including those vertex operators which can be obtained as products of (derivatives of) $b(x), c(x)$, and the matter stress tensor $T^{(\text {matter })}(x)$. $\mathcal{H}_{2}$ will contain all states of ghost number 1 carrying non-zero $k_{0}$, and also all states with $k_{0}=0$ which are obtained by the action of $b_{n}, c_{n}$ and the matter Virasoro generators on primary states of dimension $>0$ of the matter conformal field theory. Since the BRST operator $Q_{B}$ is constructed from the ghost oscillators and matter Virasoro generators, the kinetic term of the action (2.2) does not mix a state in $\mathcal{H}_{1}$ with a state in $\mathcal{H}_{2}$. A conformal transformation takes a state in $\mathcal{H}_{1}\left(\mathcal{H}_{2}\right)$ to a state in $\mathcal{H}_{1}\left(\mathcal{H}_{2}\right)$, and furthermore, the three point correlation function of two vertex operators in $\mathcal{H}_{1}$ and a vertex operator in $\mathcal{H}_{2}$ vanishes. Thus restricting the string field configuration to $\mathcal{H}_{1}$ will give a consistent truncation of the string field theory.

Since the zero momentum tachyon state described by eq.(2.8) belongs to $\mathcal{H}_{1}$, we see that switching on this tachyonic mode does not take us outside the subspace $\mathcal{H}_{1}$. In particular the tachyonic ground state will correspond to a state $\left|\Phi_{0}\right\rangle$ with no component along $\mathcal{H}_{2}$, and satisfying the equations of motion derived from the truncated action. Since integrating out all the modes in $\mathcal{H}_{1}$ other than $c_{1}|0\rangle$ may not lead to a meaningful approximation, [ , we denote by the single symbol $T$ the set of all the modes of $\mathcal{H}_{1}$, and by $\widetilde{\mathcal{S}}(T)$ the truncated string field theory action, with the string field configuration $|\Phi\rangle$ restricted to $\mathcal{H}_{1}$. Since $\mathcal{H}_{1}$ involves only those states which carry zero $X^{0}$ momentum, the inner product as well the three point function appearing in eq.(2.2) will contain a $\delta(0)$ term, representing the infinite contribution from the time integral of a time independent lagrangian. Thus the lagrangian $\widetilde{\mathcal{L}}(T)$ for this configuration can be identified as the action $\widetilde{\mathcal{S}}(T)$ with this volume factor $L=2 \pi \delta(0)$ removed. Once $\widetilde{\mathcal{L}}$ has been constructed this way, the tachyonic potential $V(T)$ can be identified with $-\widetilde{\mathcal{L}}(T)$.

\footnotetext{
${ }^{6}$ Indeed, the true ground state may not have any component along $c_{1}|0\rangle$.
} 
Computation of $V(T)$ only involves correlation functions involving the ghost fields and the matter energy momentum tensor with central charge 26. These correlation functions are completely universal. In particular, they are insensitive to all the details of the internal BCFT. As a result, $V(T)$ has a universal form for all internal BCFT except for the overall multiplicative factor $g_{o}^{-2}$ in front of the action (2.2). Thus the tachyon potential has the form:

$$
V(T)=\frac{1}{g_{o}^{2}} h(T),
$$

where $h(T)$ is some universal function independent of the choice of the internal BCFT.

We shall now show that at $T=0$ the mass of the D-brane described by the action (2.2) is related to $g_{o}^{-2}$. To see this let us consider the kinetic term in (2.2) involving the mode $\int d k_{0} \phi^{i}\left(k_{0}\right) c_{1} \alpha_{-1}^{i}\left|k_{0}\right\rangle$. Here $\alpha_{n}^{i}$ denotes the oscillator of the free world-sheet scalar field $X^{i}$, and $\left|k_{0}\right\rangle$ denotes the state $\exp \left(i k_{0} X^{0}(0)\right)|0\rangle$. Only the $c_{0} L_{0}^{\text {matter }}$ term of the BRST charge $Q_{B}$ contributes to the $k_{0}$ dependent part of the kinetic term involving this mode, and the result is given by

$$
2 \pi \frac{1}{2}\left(g_{o}\right)^{-2} \int d k_{0}\left(k_{0}\right)^{2} \phi^{i}\left(k_{0}\right) \phi^{i}\left(-k_{0}\right),
$$

in the $\alpha^{\prime}=1$ unit. If $\psi^{i}(t) \equiv \int d k_{0} e^{i k_{0} t} \phi^{i}\left(k_{0}\right)$ denotes the Fourier transform of $\phi^{i}\left(k_{0}\right)$, then the above action can be rewritten as

$$
\frac{1}{2}\left(g_{o}\right)^{-2} \int d t \partial_{t} \psi^{i} \partial_{t} \psi^{i}
$$

where $t$ denotes the time variable conjugate to $k_{0}$. Up to an overall normalization factor, $\psi^{i}$ has the interpretation of the location of the D-brane in the $x^{i}$ direction. This normalization factor may be determined as follows. Instead of taking a single D-brane, let us take a pair of identical D-branes, separated by a distance $b^{i}$ along the $X^{i}$ direction. Then each state in the open string Hilbert space carries a $2 \times 2$ Chan Paton factor, and states with off diagonal Chan Paton factors, representing open strings stretched between the two branes, are forced to carry an amount of winding charge $b^{i}$ along $X^{i}$. If we now move one of the branes by an amount $Y^{i}$ along $X^{i}$, the change in the (mass) ${ }^{2}$ of the open string with Chan Paton factors $\left(\begin{array}{ll}0 & 1 \\ 0 & 0\end{array}\right)$ and $\left(\begin{array}{ll}0 & 0 \\ 1 & 0\end{array}\right)$ should be given by:

$$
\frac{1}{(2 \pi)^{2}}\left\{(\vec{b}+\vec{Y})^{2}-\vec{b}^{2}\right\}=\frac{1}{2 \pi^{2}} \vec{b} \cdot \vec{Y}+O\left(\vec{Y}^{2}\right) .
$$


In the above equation we have used the fact that with our choice of units, the string tension is equal to $(1 / 2 \pi)$. On the other hand, since $\psi^{i}$ denotes the mode which translates the brane, moving one of the branes along $X^{i}$ will correspond to switching on a constant $\psi^{i}$. This is represented by a string field background

$$
\psi^{i} c_{1} \alpha_{-1}^{i}|0\rangle \otimes\left(\begin{array}{ll}
1 & 0 \\
0 & 0
\end{array}\right)
$$

We can now explicitly use the string field theory action (2.2) to calculate the change of the (mass) $)^{2}$ of states with Chan Paton factors $\left(\begin{array}{ll}0 & 1 \\ 0 & 0\end{array}\right)$ and $\left(\begin{array}{ll}0 & 0 \\ 1 & 0\end{array}\right)$ due to the presence of this background string field. The result is

$$
\frac{1}{\sqrt{2} \pi} \vec{b} \cdot \vec{\psi}+O\left(\vec{\psi}^{2}\right) .
$$

Comparing eqs.(2.12) and (2.14) we get

$$
\psi^{i}=\frac{Y^{i}}{\sqrt{2} \pi} .
$$

Once we have determined the relative normalization between $\psi^{i}$ and $Y^{i}$, we can return to the system containing a single brane.7 Substituting eq.(2.15) into eq.(2.11), we get,

$$
\frac{1}{2}\left(g_{o}\right)^{-2}\left(2 \pi^{2}\right)^{-1} \int d t \partial_{t} Y^{i} \partial_{t} Y^{i}
$$

This contribution to the D-brane world-volume action can be interpreted as due to the kinetic energy associated with the collective motion of the D-brane in the non-compact transverse directions. This allows us to identify the D-brane mass as

$$
M=\left(2 \pi^{2}\right)^{-1}\left(g_{o}\right)^{-2}
$$

Thus eq.(2.9) can be rewritten as

$$
V(T)=M f(T)
$$

where $f(T) \equiv 2 \pi^{2} h(T)$ is another universal function. This proves eq.(1.1) for the tachyon potential on a single bosonic D-brane.

\footnotetext{
${ }^{7}$ This can be done, for example, by moving the other brane infinite distance away by taking the limit $|\vec{b}| \rightarrow \infty$.
} 
Let us now consider the case of tachyon condensation on a brane-antibrane pair in type II string theory. Since the analysis is very similar to the case discussed above, we shall only point out the essential differences. Open string field theory with cubic action has been constructed in [7]. The string field contains two separate components, one from the Neveu-Schwarz (NS) sector and the other from the Ramond (R) sector; but for the study of tachyon potential we can set to zero the $\mathrm{R}$ sector fields. A generic NS sector string field configuration is a state in the Hilbert space $\mathcal{H}$ of the form $\Phi(0)|0\rangle$, where $|0\rangle$ denotes the $\mathrm{SL}(2, \mathrm{R})$ invariant vacuum, and $\Phi(x)$ is the product of $e^{-\phi(x)}$ with an arbitrary operator $\mathcal{O}(x)$ of ghost number 1 , made from products of (derivatives of) $b, c$, the bosonic ghost fields $\beta, \gamma$, and matter operators. The ghost charge is defined such that $b$ and $\beta$ carry ghost number -1 and $c$ and $\gamma$ carry ghost number $1 . \phi$ denotes the scalar field obtained by 'bosonizing' the $\beta-\gamma$ system 28]. In the left hand side of the normalization condition (2.6) we now need to include an additional factor of $e^{-2 \phi(0)}$ besides the $c_{-1} c_{0} c_{1}$ factor. There is a further subtlety due to the fact that $\mathcal{H}$ contains four sectors labelled by the $2 \times 2$ Chan Paton (CP) factor. We shall take the identity matrix $I$ and three Pauli matrices $\sigma_{i}$ to be the four independent CP factors. States in the CP sector $I$ and $\sigma_{3}$ satisfy the conventional GSO projection rules according to which $|0\rangle$ is even, and $e^{-\phi}$, $\beta, \gamma$ are odd. States in the CP sector $\sigma_{1}$ and $\sigma_{2}$ satisfy the opposite GSO projection rules according to which $|0\rangle$ is odd. The tachyon field is complex, but we shall restrict to configurations with real tachyon background. The zero momentum tachyon field then corresponds to the state created by the vertex operator $c(0) e^{-\phi(0)} \otimes \sigma_{1}$ on $|0\rangle$.

The string field theory action has a form very similar to (2.2), with the difference that the cubic interaction vertex also contains an insertion of the picture changing operator 28 in the correlation function. Since this operator involves only ghost fields and the superstress tensor of the matter fields, it is independent of the choice of BCFT describing the brane antibrane pair and will not affect our argument. As in the case of bosonic open string field theory, we can obtain a consistent truncation of the string field theory action by restricting $|\Phi\rangle$ to states for which the corresponding vertex operator $\Phi(x)$ is built from products of (derivatives of) the ghost fields, and the matter super stress tensor. This includes the energy momentum tensor $T^{(\text {matter })}(x)$ and the supercurrent $G^{(\text {matter })}(x)$. Furthermore since $\left(\sigma_{1}\right)^{2}$ is the identity matrix $I$, we can restrict ourselves to states with CP factors $I$ and $\sigma_{1}$ only, with the usual GSO projection on the states with CP factor $I$, and opposite GSO projection on the states with CP factor $\sigma_{1}$. The resulting truncated 
action is again universal, and in particular insensitive to the details of the internal BCFT. This shows that the tachyon potential has the form (2.9) for some universal function $h(T)$. (This of course is different from the universal function which appears in bosonic string theory.) Furthermore the mass of the D-brane is still given by an equation similar to (2.17). Thus $V(T)$ has the form given in eq.(2.18).

One of the crucial assumptions in our argument is that the BCFT describing the D-brane anti-D-brane system has a factorized form so that the conformal field theory describing the open strings is identical in each of the four CP sectors (except for opposite GSO projections in sectors $\sigma_{1}$ and $\sigma_{2}$ ). In particular, $e^{-\phi(0)} c(0)|0\rangle \otimes \sigma_{1}$ must be an allowed state in the theory. Formally this can be achieved if the antibrane is always defined to be the configuration obtained from the brane by the operation of $(-1)^{F_{L}}$, where $(-1)^{F_{L}}$ denotes the transformation which changes the sign of all the R-R and R-NS sector closed string states. In the language of boundary states this means that the antibrane is defined to have the same boundary state as the brane, except that the sign of all the RR states is reversed. However we should keep in mind that it is certainly possible to construct brane-antibrane system which does not fall into this category. A simple example would be brane-antibrane pair separated by a distance $b$ in a direction transverse to the brane. In this case the states in the CP sector $\sigma_{1}$ and $\sigma_{2}$ are forced to carry non-zero string winding charge proportional to $b$, and hence the string field configuration describing a zero momentum tachyon background is no longer of the form $c(0) e^{-\phi(0)}|0\rangle \otimes \sigma_{1}$. Instead it corresponds to a state built from a non-trivial primary state of the BCFT. Thus our argument for the universility of the tachyon potential is no longer valid in this case. A similar situation arises, for example, if either the brane or the antibrane (but not both) carries a Wilson line or a magnetic field tangential to its world volume.

A very similar argument can be given for the universality of the tachyon potential on a non-BPS D-brane of type II string theory. In fact, since the non-BPS D-brane of type IIB (IIA) string theory can be regarded as the result of modding out a brane-antibrane pair of type IIA (IIB) string theory by $(-1)^{F_{L}}[10]$, the universality of the tachyon potential on a brane-antibrane system of type II string theory automatically implies the universality of the tachyon potential on a non-BPS D-brane of type II string theory.

The analysis of this section indicates that it should be possible to describe the string field configuration corresponding to $T=T_{0}$ as a universal state in $\mathcal{H}_{1}$. This state should represent a solution of the classical equations of motion of string field theory, and should 
have the property that when we analyze small fluctuations of string field around this solution, the spectrum should not contain any physical states. (This is necessary if we are to interprete the configuration $T=T_{0}$ as the vacuum without any brane.) We should caution the reader however that our arguments are quite formal, since a priori there is no reason to expect that the $T=T_{0}$ configuration can be represented as a normalizable state in $\mathcal{H}_{1}$. Nevertheless, formal solutions of string field theory equations of motion have provided valuable insight in the past 29, 30]. In fact, ref. 30] does contain examples of such formal solutions which do not have any physical excitations. Finding a (formal) solution of the string field theory equations of motion which satisfies eq.(1.3), and hence represents the vacuum state, remains an open problem.

We end this section by noting that the result of this section has been implicitly used in ref. [4] in classifying D-branes via K-theory. Universality of the tachyon potential, together with eq.(1.3), shows that a brane and an antibrane can always annihilate via tachyon condensation as long as their boundary states differ from each other just by a change of sign of the Ramond-Ramond states. This requires that they carry the same gauge bundle, i.e. that only gauge fields with CP factor $I$ are excited. Such brane-antibrane annihilation forms a crucial ingredient in establishing one to one correspondence between stable D-branes and elements of the K-group.

\section{Fate of the U(1) gauge field under tachyon conden- sation}

In this section we shall use the results of the previous section to discuss the fate of the $\mathrm{U}(1)$ gauge field on the D-brane under tachyon condensation. The salient points of this analysis were already given in [15].

Let us begin with the bosonic D-brane. There is a U(1) gauge field living on the Dbrane. The tachyon is neutral under the gauge group; hence our intuitions from quantum field theory will tell us that the gauge fields will remain massless even when the tachyon condenses. On the other hand if $T=T_{0}$ corresponds to the vacuum without any Dbranes, as has been conjectured, then clearly there cannot be a $U(1)$ gauge field living on the brane after tachyon condensation. How do we resolve this apparent contradiction? A related question is as follows. If we consider a pair of D-branes (not necessarily of the same kind) separated by a distance, then there is an open string state with one end on 
each brane. If we now let the tachyon on one of the branes condense, then what happens to this open string state? If the $T=T_{0}$ configuration really represents the vacuum, then there cannot be an open string ending at the original location of the brane after tachyon condensation.

The resolution that we propose is as follows. We conjecture that at $T=T_{0}$ the action of the $\mathrm{U}(1)$ gauge field on the D-brane world volume is independent of the gauge field. In fact, we conjecture that the action is independent of all the massless fields living on the D-brane world volume. Thus the gauge field is no longer dynamical, but acts as an auxiliary field which forces the corresponding $U(1)$ current to vanish identically. In particular this means that open strings with one end on this brane and another end on some other brane, being charged under the $\mathrm{U}(1)$, is no longer a physical state. Physically this can be explained by saying that since effectively the U(1) gauge coupling becomes infinite, any state charged under this $\mathrm{U}(1)$ becomes infinitely massive and hence decouples from the spectrum. []

Although we have no general proof of this statement, we shall now show that our analysis of the previous section can be used to lend support to this conjecture. For this, let us start with a D-p-brane of the bosonic string theory, and compactify all directions tangential to the brane on a torus $T^{p}$ of large radii. Let $\vec{y}$ denote the directions tangential to the brane, $\left\{\varphi_{a}(\vec{y})\right\}$ denote an arbitrary time independent configuration of all massless fields living on the brane world-volume, and $T$ denote the tachyonic mode(s) discussed in the last section. We denote by $\mathcal{L}\left(\left\{\varphi_{a}(\vec{y})\right\}, T\right)$ the effective lagrangian of the brane obtained by integrating out all other modes. Note that $T$ correspond to mode(s) carrying zero momentum along the world-volume direction, whereas the massless fields $\left\{\varphi_{a}(\vec{y})\right\}$ are allowed arbitrary dependence on the world-volume coordinates. All other modes have been integrated out. This would typically give an effective lagrangian which is non-local on the D-brane world-volume, but this will not affect our discussion.

At this point we need to make some further remark about the choice of the coordinate $T$ in the configuration space. Let $\left\{\varphi_{a}^{c l}(\vec{y})\right\}$ denote some particular classical solution of the equations of motion at $T=0$. We assume that for every such classical solution there is a BCFT describing open string propagation in this background $\left\{\varphi_{a}^{c l}\right\}$. In that case, we can formulate string field theory around this new background and define a tachyonic mode around this background using the prescription of the last section. We shall choose

\footnotetext{
${ }^{8}$ This interpretation makes contact with the conjecture of ref. [14] that this $\mathrm{U}(1)$ gauge field is confined.
} 


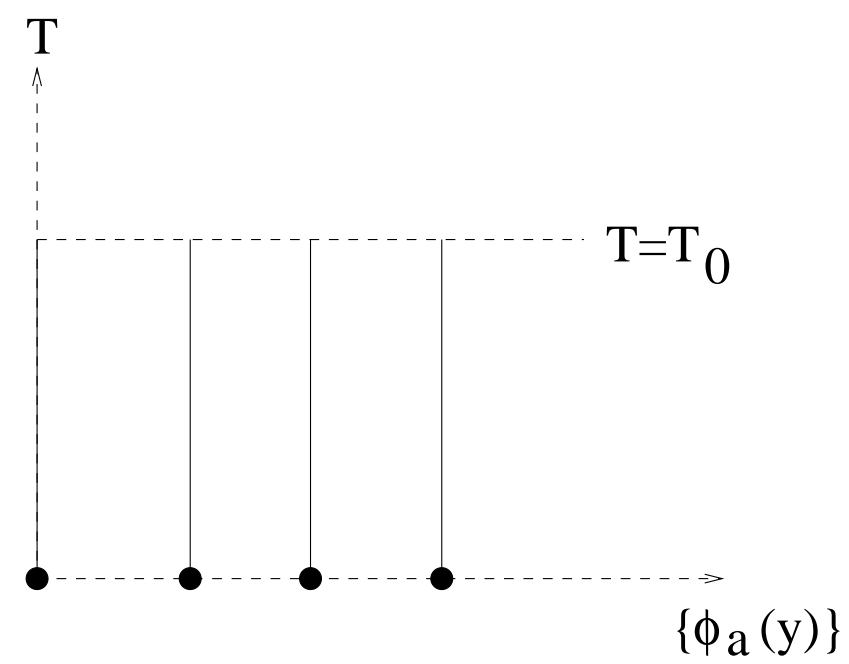

Figure 1: This diagram schematically illustrates the choice of coordinate system in the configuration space. The horizontal axis denotes the set of all time independent configurations of massless fields, and the vertical axis denotes the tachyonic mode(s) T. The black dots on the horizontal axis are the classical solutions of the equations of motion involving massless fields only. The vertical line originating from a black dot represents the effect of switching on the tachyonic mode(s) of the string field theory formulated around the BCFT associated with the particular black dot.

the coordinate $T$ appearing in $\mathcal{L}\left(\left\{\varphi_{a}(\vec{y})\right\}, T\right)$ in such a way that around every classical solution, keeping $\left\{\varphi_{a}(\vec{y})\right\}$ fixed at $\left\{\varphi_{a}^{c l}(\vec{y})\right\}$ and changing $T$ corresponds to switching on the tachyonic mode(s) of the string field theory formulated in the background $\left\{\varphi_{a}^{c l}(\vec{y})\right\}$. This has been schematically illustrated in Fig.1. In principle there could be obstruction to such a choice of coordinates; we shall assume that there is no such obstruction.

Since $\varphi_{a}(\vec{y})=0$ denotes a trivial classical solution representing the original D-brane, we have, according to eq.(1.1)

$$
\mathcal{L}\left(\varphi_{a}=0, T\right)=-M_{0} f(T)
$$

where $M_{0}$ denotes the mass of the brane for $\varphi_{a}=0$. We have chosen the additive constant in $\mathcal{L}$ such that $\mathcal{L}$ vanishes at $\varphi_{a}(\vec{y})=0, T=0$.

\footnotetext{
${ }^{9}$ This is natural from the point of view of string field theory formulated in the background BCFT corresponding to $\varphi_{a}=0, T=0$. On the other hand, from the point of view of the effective action, it is often more natural to choose this additive constant in such a way that $\mathcal{L}\left(\varphi_{a}=0, T=0\right)$ is equal to
} 
solution of the equations of motion representing a new BCFT, and $M$ denote the mass of the D-brane described by this new BCFT. section, the effective lagrangian of $T$, formulated around the new background, should be given by $-M f(T)$. This gives,

$$
\mathcal{L}\left(\varphi_{a}^{c l}, T\right)=-M f(T)+K
$$

where $K$ is an additive constant. The origin of this constant may be understood as follows. In defining effective lagrangian $\mathcal{L}$, we have fixed the additive constant in the action in such a way that the lagrangian vanishes when all the fields are set to zero. In this convention, if $\left\{\varphi_{a}^{c l}\right\}$ denotes a time independent classical solution of the equations of motion reflecting a new BCFT, then the value of the lagrangian of the original string field theory, evaluated at $\varphi_{a}=\varphi_{a}^{c l}$, will reflect the difference between the potential energies of the initial and the final configurations. On the other hand the effective lagrangian obtained by integrating out the degress of freedom of the string field theory action formulated directly around the new BCFT will have zero value when all the fields in this new string field theory action are set to zero. Thus the two effective lagrangians must differ by an additive constant $K$. It is fixed by demanding that

$$
\mathcal{L}\left(\varphi_{a}^{c l}, T=0\right)-\mathcal{L}\left(\varphi_{a}=0, T=0\right)=-\left(M-M_{0}\right)
$$

Since $f(0)=0$, this gives, using eqs.(3.1) and (3.2)

$$
K=M_{0}-M
$$

Hence

$$
\mathcal{L}\left(\varphi_{a}^{c l}, T\right)=-M(1+f(T))+M_{0}
$$

Using eqs.(3.5) and (1.3) we see that,

$$
\mathcal{L}\left(\varphi_{a}^{c l}, T_{0}\right)=M_{0} .
$$

$-M_{0},-$ the negative of the mass of the original D-brane. This is what is done, for example, in writing the action in the Born-Infeld form.

${ }^{10}$ If we consider a new BCFT with the open string coupling constant fixed, then the mass of the Dbrane does not depend on the BCFT. But it is more natural to keep the closed string coupling constant (dilaton) fixed as we change the open string background. Since the relationship between the closed and the open string coupling constant does depend on the BCFT[33], the D-brane in the new background can have a different mass. 
In other words the lagrangian at $T=T_{0}$ has the same value $M_{0}$ for all $\left\{\varphi_{a}^{c l}(\vec{y})\right\}$ which correspond to solutions of the equations of motion at $T=0$. Although this does not prove that $\mathcal{L}\left(\varphi_{a}, T_{0}\right)$ is independent of $\varphi_{a}$ (and hence in particular of the $\mathrm{U}(1)$ gauge fields) for all $\varphi_{a}$, it certainly lends support to this conjecture.

In the specific context of the $\mathrm{U}(1)$ gauge field, note that if $F_{m n}$ denote the components of the $\mathrm{U}(1)$ gauge field strength on the D-brane, then since constant $F_{m n}$ is a solution of the equations of motion and describes a BCFT, the lagrangian at $T=T_{0}$ is independent of $F_{m n}$ at least for constant $F_{m n}$. Thus at $T=T_{0}, \mathcal{L}$ can at most contain terms involving derivatives of $F_{m n}$. This establishes that $\mathcal{L}\left(F_{m n}, T_{0}\right)$ does not contain the standard gauge kinetic term since it vanishes for constant $F_{m n}$, and hence even if $\mathcal{L}$ is not completely independent of $F_{m n}$ at $T=T_{0}$, it does not represent a standard gauge theory.

The fact that $\mathcal{L}\left(F_{m n}, T_{0}\right)$ does not depend on $F_{m n}$ for constant $F_{m n}$ can also be seen via a T-duality transformation, starting with the assumption that at $F_{m n}=0$ the mass of the brane, $-\mathcal{L}+M_{0}$, vanishes at the extremum $T_{0}$ of the tachyon potential. For this let $x^{1}$ and $x^{2}$ denote two of the directions tangential to the D-brane which have been compactified. For $F_{m n}=0$, an $R \rightarrow(1 / R)$ duality transformation along the $x^{2}$ direction converts this D-brane to a D-brane with Dirichlet boundary condition along the $x^{2}$ direction, and Neumann boundary condition along the $x^{1}$ direction. Since the mass of the brane does not change under T-duality, the mass of the T-dualized brane, and hence also its tension, vanishes at the extremum $T_{0}$ of the tachyon potential. Now if we switch on the constant field strength $F_{12}$ in the original D-brane, it corresponds to putting Dirichlet boundary condition on some linear combination of $x^{1}$ and $x^{2}$ in the T-dual description. Thus we effectively change the orientation of the brane in the T-dual description. But if the tension of this D-brane vanishes at some extremum of the tachyon potential, it continues to vanish even if we change the orientation of the brane, and hence the total mass of the brane still vanishes. But this is equal to the mass of the original brane at constant $F_{12}$ and $T=T_{0}$, i.e. to $-\mathcal{L}\left(F_{12}, T_{0}\right)+M_{0}$. Thus we see that $\mathcal{L}\left(F_{12}, T_{0}\right)=M_{0}$, i.e. it is independent of $F_{12}$.

This analysis can be easily generalized to the case of the brane-antibrane system and the non-BPS D-brane of type II string theories. In carrying out this analysis one should keep in mind that for the brane-antibrane system, the $\mathrm{U}(1)$ which must be switched on is the diagonal combination of the two U(1)'s on the brane and the antibrane (corresponding to $\mathrm{CP}$ sector $I$ ) so that the new BCFT satisfies the conditions for validity of our analysis. It is only for this $U(1)$ that we conjecture that the action is independent of the gauge field 
at the minimum of the tachyon potential. The other $\mathrm{U}(1)$ gauge symmetry is broken due to Higgs mechanism in the presence of a non-vanishing vev of the tachyon field.

\section{Tachyon potential in closed bosonic string theory}

We can repeat our analysis for the tachyon of closed bosonic string theory in arbitrary conformal field theory background. In this case a string field configuration is represented by an arbitrary state $|\Phi\rangle$ in the closed string Hilbert space carrying ghost number 2, and satisfying the condition

$$
\left(b_{0}-\bar{b}_{0}\right)|\Phi\rangle=0, \quad\left(L_{0}-\bar{L}_{0}\right)|\Phi\rangle=0
$$

There is an action similar to (2.2) for the closed string field theory, with the difference that the action is non-polynomial 31, 32, involving quartic and higher order vertices. However, each of these vertices are constructed from conformal field theory correlation functions in a manner analogous to (2.2). Thus we can find a consistent truncation of the theory by restricting the string field configuration to a subspace $\mathcal{H}_{1}$ built from $|0\rangle$ by the action of the ghost oscillators and the matter Virasoro generators.

The zero momentum tachyon corresponds to the state $c_{1} \bar{c}_{1}|0\rangle$, and hence is an element of $\mathcal{H}_{1}$. Thus starting from the truncated action and integrating out the other fields we can recover the tachyon potential.⿴囗十 This is insensitive to the details of the conformal field theory on which the bosonic string theory is based, and thus is universal. However, unlike in the case of open string tachyons, in this case there is no compelling reason to believe that there exists a non-trivial classical solution of the string field theory equations of motion in this truncated theory; hence the physical significance of the tachyon potential obtained this way is not entirely obvious.

Acknowledgement: I wish to thank A. Dabholkar and B. Zwiebach for useful discussions.

\section{References}

\footnotetext{
${ }^{11}$ In this case the zero momentum massless dilaton, corresponding to the state $\left(c_{-1} c_{1}-\bar{c}_{-1} \bar{c}_{1}\right)|0\rangle$ also belongs to the set $\mathcal{H}_{1}$ and cannot be integrated out. Thus by this procedure we shall get the potential involving the tachyon and the zero momentum dilaton.
} 
[1] A. Sen, "Stable non-BPS bound states of BPS D-branes," JHEP 08, 010 (1998) hep-th/9805019.

[2] A. Sen, "Tachyon condensation on the brane antibrane system," JHEP 08, 012 (1998) hep-th/9805170.

[3] A. Sen, "SO(32) spinors of type I and other solitons on brane-antibrane pair," JHEP 09, 023 (1998) hep-th/9808141.

[4] E. Witten, "D-branes and K-theory," JHEP 12, 019 (1998) hep-th/9810188.

[5] P. Horava, "Type IIA D-branes, K-theory, and matrix theory," Adv. Theor. Math. Phys. 2, 1373 (1999) hep-th/9812135.

[6] E. Witten, "Noncommutative Geometry And String Field Theory," Nucl. Phys. B268, 253 (1986).

[7] E. Witten, "Interacting Field Theory Of Open Superstrings," Nucl. Phys. B276, 291 (1986).

[8] O. Bergman and M.R. Gaberdiel, "Stable non-BPS D-particles," Phys. Lett. B441, 133 (1998) hep-th/9806155.

[9] A. Sen, "Type I D-particle and its interactions," JHEP 10, 021 (1998) hepth/9809111.

[10] A. Sen, "BPS D-branes on non-supersymmetric cycles," JHEP 12, 021 (1998) hepth/9812031.

[11] A. Sen, "Descent relations among bosonic D-branes," hep-th/9902105.

[12] A. Recknagel and V. Schomerus, "Boundary deformation theory and moduli spaces of D-branes," Nucl. Phys. B545, 233 (1999) hep-th/9811237;

C.G. Callan, I.R. Klebanov, A.W. Ludwig and J.M. Maldacena, "Exact solution of a boundary conformal field theory," Nucl. Phys. B422, 417 (1994) hep-th/9402113;

J. Polchinski and L. Thorlacius, "Free fermion representation of a boundary conformal field theory," Phys. Rev. D50, 622 (1994) hep-th/9404008.

[13] M. Srednicki, "IIB or not IIB," JHEP 08, 005 (1998) hep-th/9807138. 
[14] P. Yi, "Membranes from five-branes and fundamental strings from Dp branes," Nucl. Phys. B550, 214 (1999) hep-th/9901159.

[15] A. Sen, "Supersymmetric world-volume action for non-BPS D-branes," JHEP 10, 008 (1999) hep-th/9909062.

[16] K. Bardakci, Nucl. Phys. B68 (1974) 331; B70 (1974) 397; B133 (1978) 297.

[17] M.B. Green, "Pointlike states for type 2b superstrings," Phys. Lett. B329, 435 (1994) hep-th/9403040.

[18] T. Banks and L. Susskind, "Brane - Antibrane Forces," hep-th/9511194.

[19] I. Pesando, "On the effective potential of the Dp Dp-bar system in type II theories," Mod. Phys. Lett. A14, 1545 (1999) hep-th/9902181.

[20] V.A. Kostelecky and S. Samuel, "The Static Tachyon Potential In The Open Bosonic String Theory," Phys. Lett. B207, 169 (1988); "On A Nonperturbative Vacuum For The Open Bosonic String," Nucl. Phys. B336, 263 (1990).

[21] V.A. Kostelecky and S. Samuel, "Collective Physics In The Closed Bosonic String," Phys. Rev. D42, 1289 (1990).

[22] A. Belopolsky and B. Zwiebach, "Off-shell closed string amplitudes: Towards a computation of the tachyon potential," Nucl. Phys. B442, 494 (1995) hep-th/9409015.

[23] A. Belopolsky, "Effective Tachyonic potential in closed string field theory," Nucl. Phys. B448, 245 (1995) hep-th/9412106.

[24] T. Banks, "The Tachyon potential in string theory," Nucl. Phys. B361, 166 (1991).

[25] B. Zwiebach, "Oriented open-closed string theory revisited," Annals Phys. 267, 193 (1998) hep-th/9705241.

[26] A. LeClair, M.E. Peskin and C.R. Preitschopf, "String Field Theory On The Conformal Plane. 1. Kinematical Principles," Nucl. Phys. B317, 411 (1989); "String Field Theory On The Conformal Plane. 2. Generalized Gluing," Nucl. Phys. B317, 464 (1989). 
[27] A. Sen, "Open String Field Theory In Nontrivial Background Field: Gauge Invariant Action," Nucl. Phys. B334, 350 (1990); "Open String Field Theory In Arbitrary Background Field. 2. Feynman Rules And Four Point Amplitudes," Nucl. Phys. B334, 395 (1990); "Open String Field Theory In Nontrivial Background Field. 3. N Point Amplitude," Nucl. Phys. B335, 435 (1990).

[28] D. Friedan, E. Martinec and S. Shenker, "Covariant Quantization Of Superstrings," Phys. Lett. 160B, 55 (1985).

[29] G.T. Horowitz, J. Lykken, R. Rohm and A. Strominger, "A Purely Cubic Action For String Field Theory," Phys. Rev. Lett. 57, 283 (1986).

[30] G.T. Horowitz, J. Morrow-Jones, S.P. Martin and R.P. Woodard, "New Exact Solutions For The Purely Cubic Bosonic String Field Theory," Phys. Rev. Lett. 60, 261 (1988).

[31] M. Saadi and B. Zwiebach, "Closed String Field Theory From Polyhedra," Ann. Phys. 192, 213 (1989).

[32] B. Zwiebach, "Closed string field theory: An Introduction," hep-th/9305026.

[33] N. Seiberg and E. Witten, "String theory and noncommutative geometry," JHEP 09, 032 (1999) hep-th/9908142. 